\title{
Structural and Ultrastructural Morphological Evaluation of Giant Anteater (Myrmecophaga Tridactyla) Prostate Gland
}

Fernanda B C de Moura

Sao Paulo State University

Letícia H. T. S. Sampaio

Sao Paulo State University

Priscila E. Kobayashi

Sao Paulo State University

Renee Laufer-Amorim

Sao Paulo State University

João Carlos P. Ferreira

Sao Paulo State University

Tatiane T. N. Watanabe

North Carolina State University

Carlos E. Fonseca-Alves ( $\square$ carlos.e.alves@unesp.br)

Sao Paulo State University

\section{Research Article}

Keywords: Xenarthra, Morphology, Immunohistochemistry, Reproduction, Conservation

Posted Date: February 9th, 2021

DOl: https://doi.org/10.21203/rs.3.rs-195228/v1

License: (c) (i) This work is licensed under a Creative Commons Attribution 4.0 International License. Read Full License 


\section{Abstract}

The giant anteater (Myrmecophaga tridactyla) is a vulnerable species from Central and South Americas, that is considered possibly extinct in Belize, Guatemala, El Salvador, and Uruguay. Due to the species conservations and reproduction's importance, this research aimed to characterize the morphology, histochemical, immunohistochemical, and ultrastructural feature of the giant anteater prostate gland. For this, we collected 11 giant anteater prostate glands and performed macroscopic, morphological, histochemical, immunohistochemical, and ultrastructural analysis. Nine-prostate glands from adult and two from young subjects were studied. Grossly, the adult giant anteater prostate gland is divided in two distinct zones; the central zones composed mainly of ducts and the peripheral zones of acini formed by secretory cells. The secretory cells showed positive periodic acid-Schiff staining. Furthermore, the immunohistochemical characterization revealed a similar human prostate pattern, with p63 staining basal cells, UPIII superficial cells of prostatic urethra, AR expressing nucleus of secretory and stromal cells, and PSA staining prostatic epithelial cells. Overall, our research provided an indepth morphological description of the giant anteater prostate gland, providing a valuable information for futures studies focused on giant anteater conservation.

\section{Introduction}

The giant anteater (Myrmecophaga tridactyla), also known as the ant bear, belongs to the Myrmecophagidae family and can only be found in the tropical and subtropical areas of Central and South America ${ }^{[1]}$. The giant anteater is considered possibly extinct in Belize, Guatemala, El Salvador, and Uruguay. Overall, it is considered "vulnerable" on the International Union for Conservation of Nature (IUCN) Red List of Threatened Species ${ }^{[1]}$. Several factors contribute to population reduction and even extinction of the giant anteater, including predatory hunting, forests burnings and lack of governmental programs for conservation ${ }^{[2,3]}$. Ongoing intensive growth of agricultural activities has a critical economic role in many countries in South America; however, programs that encourage the awareness and protection of vulnerable species are still scarce ${ }^{[4]}$. Since 2019 , Brazil is the fourth country in the world with the higher number of mammals included in the IUCN red list ${ }^{[5]}$. In addition, the giant anteater has a slow reproductive cycle in nature, since they present long gestations periods - being born one cub per year - and the type of parental care can also contribute to the species decline $e^{[6]}$.

Even though the giant anteater is considered a "vulnerable" species, reported studies regarding the reproductive aspects and its association with conservation programs and development of protocols emphasizing new reproductive biotechnologies for this species are extremely scarce. ${ }^{[7]}$ Although the number of sexual accessory glands varies from different mammals, the prostate gland is present in all mammal species and it is considered one of the most important ${ }^{[8]}$.

Male dogs and male human beings are the species with the most detailed prostatic studies with complete and broad information that include full organ description based on its macroscopy, histology, and 
immunohistochemical patterns ${ }^{[8]}$. Among the immunohistochemical markers used in veterinary medicine, prostatic specific antigen (PSA), p63, uroplakin III (UPIII), and androgen receptor (AR) were already welldescribed especially in dogs ${ }^{[9]}$. To our knowledge, a complete prostatic characterization in giant anteater is yet to be investigated. Therefore, this research aimed to characterize the prostate gland in giant anteater by morphological, histochemical, immunohistochemical, and ultrastructural to aid the conservation program to this species in future.

\section{Results}

\subsection{Animals}

Thirteen free-living giant anteaters were referred to the Wild Animal Service at the Veterinary Teaching Hospital at São Paulo State University after a history of blunt trauma secondary to wildlife-vehicle collisions between January 2017 and December 2019. After postmortem examinations, 11 prostatic samples from adults (>2-year-old) and two prostatic samples from young (<2-year-old) were macroscopically and microscopically assessed. The age category was based on the total body weight and crump-to-rump length.

\subsection{Macroscopic analysis, microscopic assessment and histochemical evaluation}

The giant anteater encapsulated prostate is located dorsally to the urinary bladder and ventrally to the rectum (Fig 1).

Based on the macroscopic findings of the prostate gland, we proposed two different anatomical zones: central and peripheral zones. The central zone is located very centrally in a transversal cut of section and represents the prostatic parenchyma that surrounds the urethra (Fig 2). The peripheral zones are located at the periphery of the gland and have two grossly distinct lobes without any bridging communication between each other: right and left lobes. Both lobes are replete with gelatinous transparent fluid within multiple cavity foci (Fig 2). Interestingly, the two young subjects had no clear distinction between the peripheral prostatic lobes (Fig 2.).

Microscopically, the prostatic capsule is composed of loose vascular connective tissue some arteries, veins, nervous ganglions and some muscles bundles (Fig 3). Thus, the histological findings provided strong support for the giant anteater prostate gland's proposed division into these two distinct zones. In the central zone, it was visualized fibromuscular stroma filled with the prostatic ducts lined by stratified columnar epithelium and the prostatic urethra, characterized by transitional epithelium (Fig 3). In Masson trichrome stain, it was possible to identify the collagen and muscle bundles, staining in blue and red, respectively. The ducts epithelial cells were also positive for Periodic acid-Schiff (PAS) stain (Fig 4). In the peripheral zones, there was a stromal fibrous connective tissue with linear fibroblast, arterioles, and venules adjacent to the glycogen-rich acini, which are composed of a pseudostratified columnar epithelium. We observed that the collagen fibers, blue colored with the Masson trichrome stain, and the glycogen-rich epithelial cells were positive for PAS stains (Fig 4). 
The peripheral zone of the young subjects had $50 \%$ fewer numbers of acini when compared to the adult (Fig 3). Furthermore, the central zone in the young category did not shown any macroscopic differences when compared to the adult, however, there was lower numbers of prostatic ducts histologically (Fig 3).

\subsection{Scanning electron microscopy}

In the scanning electron microscopy, it was possible to observe a capsule surrounding the prostate gland, the central urethral area and two prostatic lobes regions composed of collagen fibers (Fig 5). In the peripherical lobes, it was observed acinar structures, and their epithelium, whereas in the central zone, it was possible to visualize the prostatic ducts around the urethra (Fig 5).

\subsection{Immunohistochemistry}

Androgen receptor (AR) showed intense positive nuclear staining in all prostatic epithelial cells (ducts and acini) and low/absent nuclear expression in the prostatic stromal cells in this species (Fig 4). Immunolabelling anti-PSA was moderate cytoplasmic in all acinar and ductal cells (Fig 4). We observed moderate nuclear P63 expression forming a continuous basal cell layer in the duct and acini of giant the anteater prostate gland (Fig 4). Furthermore, it was also seen membranous uroplakin III strong positive expression on superficial urothelial cells (umbrella cells) in the prostatic urothelial epithelium (Fig 4).

\section{Discussion}

A few studies analyze the giant anteater's reproductive tract, making it challenging to develop new strategies for this species conservation. A previous study demonstrated the sequential ejaculate fractions in giant anteaters submitted to electrojaculation which is composed of two different sequential fractions

[10]. Study in lesser anteater (Tamandua tetradactyla) describing the gross histopathological morphology of the male genital tract has also been reported. However, to the best of our knowledge, no previous studies describing the structural and ultrastructural morphology of giant anteater prostate gland has been published to this date.

The macroscopical and morphological findings date from this study described herein strongly support our proposed division and classification of the giant anteater prostate gland in two different zones: central and peripheral, similarly described in humans ${ }^{8}$. The criterion supporting the prostate gland division into central and peripheral zones is the histologic differences between zone's stroma and parenchyma, including the number of vessels, percentage of ducts and acini, and presence of striated muscle in the central zone. To our knowledge, this is a novel prostatic classification in giant anteaters and can be considered one of the differences found between this specie and its Xenarthra parent, the lesser anteater ${ }^{[7]}$.

The immunohistochemistry characterization of the great anteater prostate gland allowed us to compare our findings with human and canine ${ }^{[11]}$. Human, canine and giant anteater prostate present some similarities, including the positive expression for AR, PSA, P63, and UPIII. Interestingly, the giant anteater 
prostate gland has a continuous basal cell layer similarly seen in humans, however it differs from dogs since there is a discontinuous basal cell layer ${ }^{[8]}$.

Furthermore, the androgen receptors and the PSA markers showed a positive expression in luminal prostatic cells. On the other hand, the transitional cells from the prostatic urethra showed immunoreaction anti UPIII. Thus, the expression pattern of the prostate markers is typical among giant anteater humans and dogs $[8,9]$.

It is important to emphasize that we observed that the giant anteater's prostatic stroma has striated muscle bundles, in Masson's trichrome stain. Usually, the prostate gland of different mammals presents only smooth muscle fiber. The presence of striated muscle fibers suggests that the giant anteater prostate gland may have a voluntary muscle contraction control system. Another histochemical technique used, the PAS stain, allowed us to visualize the glycogen presents in the prostatic acini and ducts.

Finally, it is essential to emphasize that the giant anteater is a vulnerable species, which nowadays suffers constant losses in its population. The mains contribution reason for this decrease is the increasing human presence in their habitats and their slow reproductive cycle $\mathrm{e}^{[1,3,4]}$. The particularities characteristics of the giant anteater prostate study, such as morphological variations between young and adult subjects' characterization of the zones and, especially, the presence of striated musculature in the central zone raise hypothesis for possible autonomous control of the prostatic fluid ejaculate, as observed in human and canine prostates ${ }^{[12]}$. Additionally, it is possible to speculate the importance of this findings involving the participation of this gland in the giant anteater's fertility. Therefore, further studies involving the description of the prostatic innervation and the other accessory glands are welcomed to develop conservation and breeding programs.

\section{Material And Methods}

\subsection{Ethical approval}

Three independent Brazilian committees approved this study: the National Management System for Genetic Heritage and Associated Traditional Knowledge (\#A1B0E09), Chico Mendes Institute for Biodiversity Conservation (\#65487) and the ethics committee on the use of animals in research (CEUA) at Sao Paulo State University (\#0002/2018). All postmortem sampling collection was done at the Department of Veterinary Pathology Service from Sao Paulo State University (UNESP).

\subsection{Samples and morphological analysis}

\subsubsection{Samples collection}

Thirteen free-living giant anteaters were autopsied within six hours interval after death due to blunt trauma force. Fresh prostate glands initially were macroscopically evaluated based on anatomical 
measurements and organ weight. For microscopic examination, the prostatic tissue samples and testis were fixed in neutral-buffered $10 \%$ formalin for 24 hours, embedded in paraffin, and routinely processed for histologic examination (FFPE). Additionally, samples were fixed in pentane - 1,5-dial glutaraldehyde for 24 hours at $4^{\circ} \mathrm{C}$ for scanning electron microscopy. The individual subjects' information, including body weight and prostate measurement, are shown in Table 1.

Table 1. Giant anteaters autopsied information.

\begin{tabular}{|c|c|c|c|c|c|}
\hline \multirow[t]{2}{*}{ Identification } & \multirow[t]{2}{*}{ Age } & \multirow{2}{*}{$\begin{array}{l}\text { Weight } \\
(\mathrm{Kg})\end{array}$} & \multirow{2}{*}{$\begin{array}{l}\text { Central Zone } \\
\text { Size* }^{\star}\end{array}$} & \multicolumn{2}{|l|}{ Peripheral zone } \\
\hline & & & & $\begin{array}{l}\text { Left Zone Size } \\
\text { (cm) }\end{array}$ & $\begin{array}{l}\text { Right Zine Size } \\
\text { (cm) }\end{array}$ \\
\hline Subject 1 & Young & 19 & $3.1 \times 3.5 \times 1.9$ & $3.5 \times 4.1 .3 .1^{*}$ & NA \\
\hline Subject 2 & Young & 22 & $3.9 \times 3.2 \times 2.5$ & $3.9 \times 4.0 \times 2.9 *$ & NA \\
\hline Subject 3 & Adult & 38 & $5.6 \times 6.1 \times 4.3$ & $7.1 \times 3.7 \times 5.6$ & $7.2 \times 3.5 \times 5.4$ \\
\hline Subject 4 & Adult & 40 & $6.1 \times 5.4 \times 4.9$ & $6.9 \times 4.1 \times 5.4$ & $6.7 \times 4.4 \times 5.6$ \\
\hline Subject 5 & Adult & 37 & $5.6 \times 5.5 \times 4 \times 2$ & $7.3 \times 4.7 \times 5.1$ & $7.1 \times 4.9 \times 5.1$ \\
\hline Subject 6 & Adult & 35 & $5.4 \times 4.9 \times 4.1$ & $5.9 \times 2.7 \times 4.9$ & $6.2 \times 3.1 \times 4.8$ \\
\hline Subject 7 & Adult & 34 & $5.8 \times 5.1 \times 3.9$ & $6.1 \times 4.3 \times 4.8$ & $6.3 \times 4.2 \times 4.7$ \\
\hline Subject 8 & Adult & 37 & $5.6 \times 4.9 \times 4.1$ & $6.5 \times 4.1 \times 5.0$ & $6.3 \times 4.1 \times 5.1$ \\
\hline Subject 9 & Adult & 38 & $5.9 \times 3.9 \times 3.8$ & $6.8 \times 4.0 \times 4.9$ & $6.9 \times 4.2 \times 4.7$ \\
\hline Subject 10 & Adult & 39 & $6.1 \times 5.7 \times 4.5$ & $7.3 \times 4.2 \times 5.3$ & $7.1 \times 4.4 \times 5.2$ \\
\hline Subject 11 & Adult & 33 & $5.3 \times 5.1 \times 5.2$ & $6.5 \times 2.9 \times 5.0$ & $6.6 \times 2.9 \times 4.8$ \\
\hline Subject 12 & Adult & 38 & $5.4 \times 4.9 \times 4.7$ & $6.9 \times 4.7 \times 4.2$ & $7.0 \times 4.7 \times 4.5$ \\
\hline Subject 13 & Adult & 39 & $5.7 \times 5.1 \times 4.9$ & $7.1 \times 3.3 \times 5.0$ & $7.1 \times 3.3 \times 5.0$ \\
\hline
\end{tabular}

The sexual maturity estimation of studied free-living giant anteaters was based on the criteria reported by Smith (2007), Baugher (2004), and Jerex and Haloy (20003) resulting into two studied groups: adult vs young. In brief, the young category includes animals with at least 10-month-old, when the female halts are still carrying it in her back up to two-year-old, before the sexual maturity be reached. The adult category includes males with $\geq 30 \mathrm{~kg}$ of body weight associated with sexual maturity. The testicular maturity was determined by the presence of spermatogenesis in FFPE testis tissue samples. 


\subsubsection{Histochemistry}

All the histological sections were stained with hematoxylin and eosin (H\&E), Masson's trichrome stain, and Periodic acid-Schiff stain (PAS). Morphological analysis of the giant anteater's prostatic gland followed the standards established by Rossi et al. (2013) ${ }^{7}$.

\subsubsection{Scanning electron microscopy}

The prostate sample selected for scanning electron microscopy analysis (MEV-EDX, FEI Company, Quanta 200 e EDX da Oxford, 51-XMX1119) was previously fixed in pentane - 1,5-dial glutaraldehyde for 24 hours at $4^{\circ} \mathrm{C}$.

\subsubsection{Immunohistochemistry}

The tissue sections obtained from the FFPE blocks were placed on charged slides (Starfrost, Knitell, Bielefeld, Germany). For the antigen retrieval, the slides were incubated in a citrate solution $(\mathrm{pH} 6,0)$ in a pressure cooker (Pascal, Dako, Carpinteria, CA, EUA) for 45 minutes. Endogenous peroxidase was blocked by hydrogen peroxide $8 \%$ diluted in methyl alcohol for 15 minutes and treated with skim milk $8 \%$, at room temperature. The sections were incubated overnight at $4^{\circ} \mathrm{C}$ with primary antibodies anti-AR (1:50; clone ab77557; Abcam), anti-PSA (1:1500; polyclonal; Bioss), anti-P63 (1:100; clone 4A4; Dako, Carpinteria, USA) and anti-uroplakin III (UPIII; 1: 50; clone AU1, Researches technologies). A polymer detection system (Envision, Dako, Carpinteria- USA) was applied as a secondary antibody, and immunoreactive cells were visualized by colorimetric detection (3,3囚- diaminobenzidine). The sections were counterstained with Harris hematoxylin (Dinamica-Diadema, Brazil). A canine prostate was used as a positive control for all antibodies. Negative controls were applied to one section of canine prostate

The immunostaining evaluation for all antibodies was classified as negative or positive expression in the membrane, cytoplasm, or nuclear immunolabelling.

\section{Declarations}

\section{Data availability}

Simulation files and results can be obtained from the corresponding author upon reasonable request.

\section{Acknowledgements}

We want to thank the Faculty of Veterinary Medicine and Zootechny (FMVZ) from São Paulo State University (UNESP) as well as the Clinical Department of the same institution for the support and encouragement for the performance of this study.

\section{Author contributions}

The authors who participate in this research were all participative and essential for this study. 
Department of Clinic School of Veterinary Medicine and Animal Science of São Paulo State University UNESP, Botucatu, 18618-81, Brazil.

Priscila E. Kobayashi, Renee Laufer-Amorim

Department of Veterinary Surgery and Animal Reproduction School of Veterinary Medicine and Animal Science of São Paulo State University - UNESP, Botucatu, 18618-81, Brazil.

Fernanda B. C. de Moura, Letícia H. T. S. Sampaio, João Carlos P. Ferreira, Carlos E. Fonseca-Alves.

Department of Population and Health Pathobiology, College of Veterinary Medicine, North Carolina State University, Raleigh, NC, 24607, USA.

Tatiane T. N. Watanabe3

Institute of Health Sciences, Paulista University - UNIP, Bauru, 17048-290, Brazil.

Carlos E. Fonseca-Alves

Contributions

F.B.C.M and C.F.E.F.A wrote the research project and performed the study. L.H.T.S.S, P.E.K, T.T.N.W, J.C.P.F and R.L.M helped in the interpretation of results. All authors participated on the writing and reviewing the manuscript.

Corresponding author

Correspondence to Carlos E. Fonseca-Alves

Additional information

Competing interests

The authors declare no competing interests.

\section{References}

1. Miranda, F., Bertassoni, A. \& Abba, A. M. Myrmecophaga tridactyla. The IUCN Red List of Threatened Species. 10.2305/IUCN.UK (2014).

2. Medri, Í. M., Mourão, G. de M. \& Harada, A. Y. Edentata: Dieta de Tamanduá-bandeira (Myrmecophaga tridactyla) no Pantanal da Nhecolândia, Brasil (ed. Fonseca, G. A. B. et al.) 14134411 (2003).

3. Brazil's new president adds to global threat to science. Nature vol. 563 5-6 (2018). 
4. Vidolin, G. P. et al. Planos de Conservação para Espécies de Mamíferos Ameaçados (ed. IAP/Projeto Paraná Biodiversidade). 0-316 (2009).

5. IUCN Red List of Threatened Species. Choice reviews online 49 ISSN 2307-8235(2012).

6. Miranda, F.R. et al. Avaliação do Risco de Extinção de Myrmecophaga tridactyla Linnaeus, 1758 no Brasil, Processo de avaliação do risco de extinção da fauna brasileira. Preprint at ICMBio: http://www.icmbio.gov.br/portal/biodiversidade/fauna-brasileira/lista-de-especies/7049-mamiferosmyrmecophaga-tridactyla-tamandua-bandeira.html (2015).

7. Rossi, L. F. et al. Anatomy and histology of the male reproductive tract and spermatogenesis fine structure in the lesser anteater (Tamandua Tetradactyla, Myrmecophagidae, Xenarthra): Morphological evidences of reproductive functions. Journal of Veterinary Medicine Series C: Anatomia Histologia Embryologia 42, 247-256 (2013).

8. Fernando Leis-Filho, A. \& E. Fonseca-Alves, C. Anatomy, Histology, and Physiology of the Canine Prostate Gland, Veterinary Anatomy and Physiology doi:10.5772/intechopen.81410 (2018).

9. Fonseca-Alves, C. E. et al. Immunohistochemical panel to characterize canine prostate carcinomas according to aberrant p63 expression. PLoS ONE 13, https://doi.org/10.1371/journal.pone.0199173 (2018).

10. Luba, C. do N. et al. Semen characteristics and refrigeration in free-ranging giant anteaters (Myrmecophaga tridactyla). Theriogenology 84, 10.1016/j.theriogenology.2015.07.041 (2015).

11. Sun, F., Báez-Díaz, C. \& Sánchez-Margallo, F. M. Canine prostate models in preclinical studies of minimally invasive interventions: Part I, canine prostate anatomy and prostate cancer models. Translational Andrology and Urology 6, http://dx.doi.org/10.21037/tau.2017.03.61 (2017).

12. McVary, K. T., McKenna, K. E. \& Lee, C. Prostate innervation. The Prostate Suplement 8, 2-13 (1998).

\section{Figures}




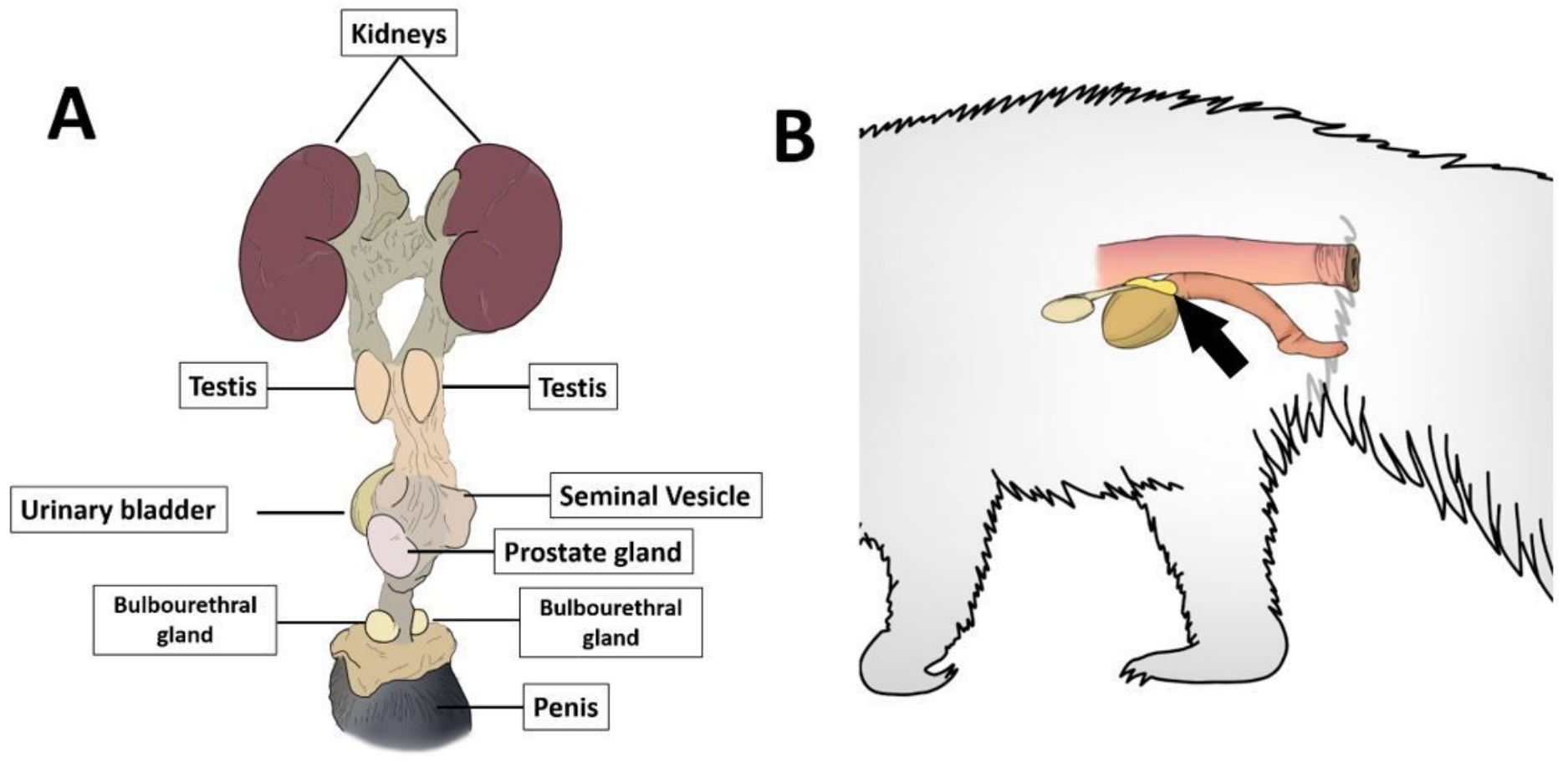

Figure 1

Schematic representation of anatomic localization of giant anteater prostate gland. A: Representation of the prostate gland dorsal do the urinary bladder (and ventral to the rectum. B: Schematic representation of the giant anteater urogenital tract showing the intracavitary testicles cranial to the urinary bladder, in which is possible to observe dorsally the prostate (arrow). 


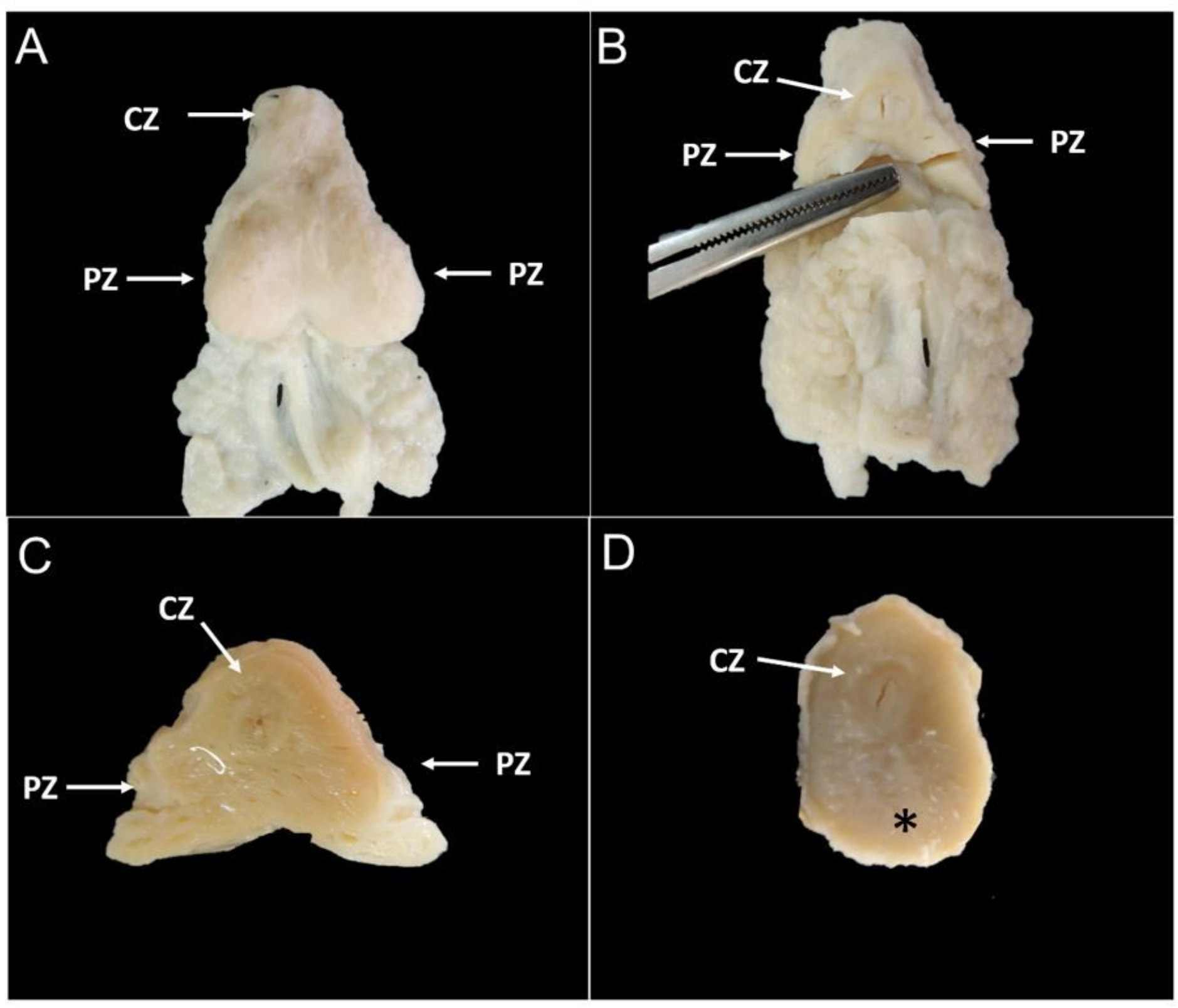

\section{Figure 2}

Gross image of adult (A-C) and young (D) giant anteater prostate glands. A: It is possible to observe a bilobulated gland with a fibrous capsule. B: Transverse section showing a central zone surrounding the prostatic urethra and the two peripheral lobes (peripheral zones). C: Macroscopic appearance of the giant anteater prostate gland, with the peripheral zones characterized by the presence of several cavities filled with a gelatinous material (arrow) D: Transverse section showing a central prostatic urethra surrounded by a central zone and no clear definition of the peripheral zones (asterisk). CZ: central zone; PZ: peripheral zone. 


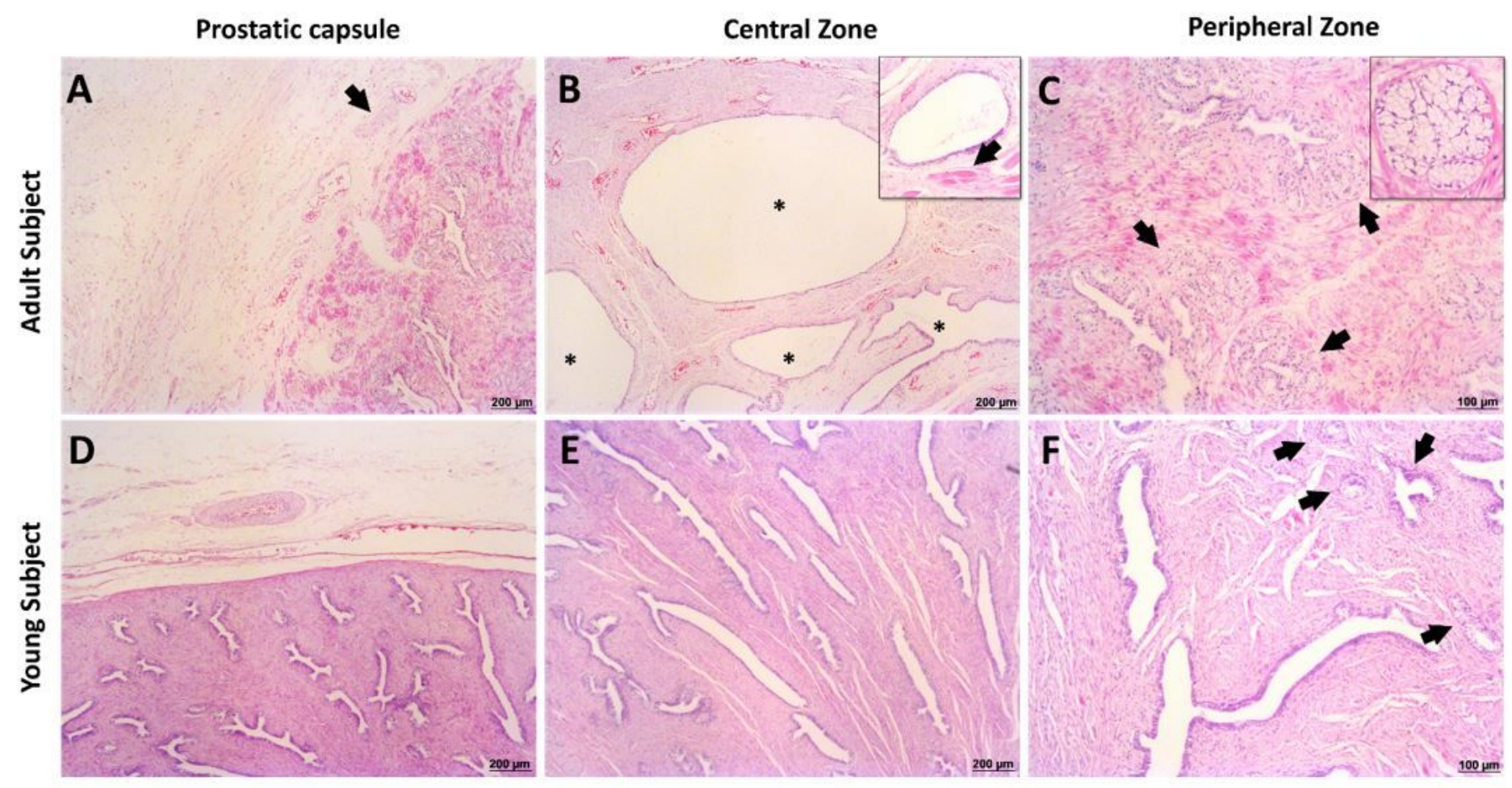

Figure 3

Histological evaluation of Giant Anteater prostate gland. A: peripheral region of the prostate gland capsule of an adult subject presenting the peripheral nerves (arrow) and blood vessels. B: Central zone of the adult Gian Anteater, presenting prostatic ducts (insert) with large lumen (asterisk). C: peripheral zone showing a central duct surrounded by secretory cells (insert). D: peripheral region of a young Giant Anteater capsule showing large caliber blood vessels. E: central zone of a young Giant Anteater enriched by ducts. F: Peripheral zone of a young Giant Anteater prostate gland present high caliber ducts and small cluster with secretory cells (arrows). Hematoxylin and eosin staining. 

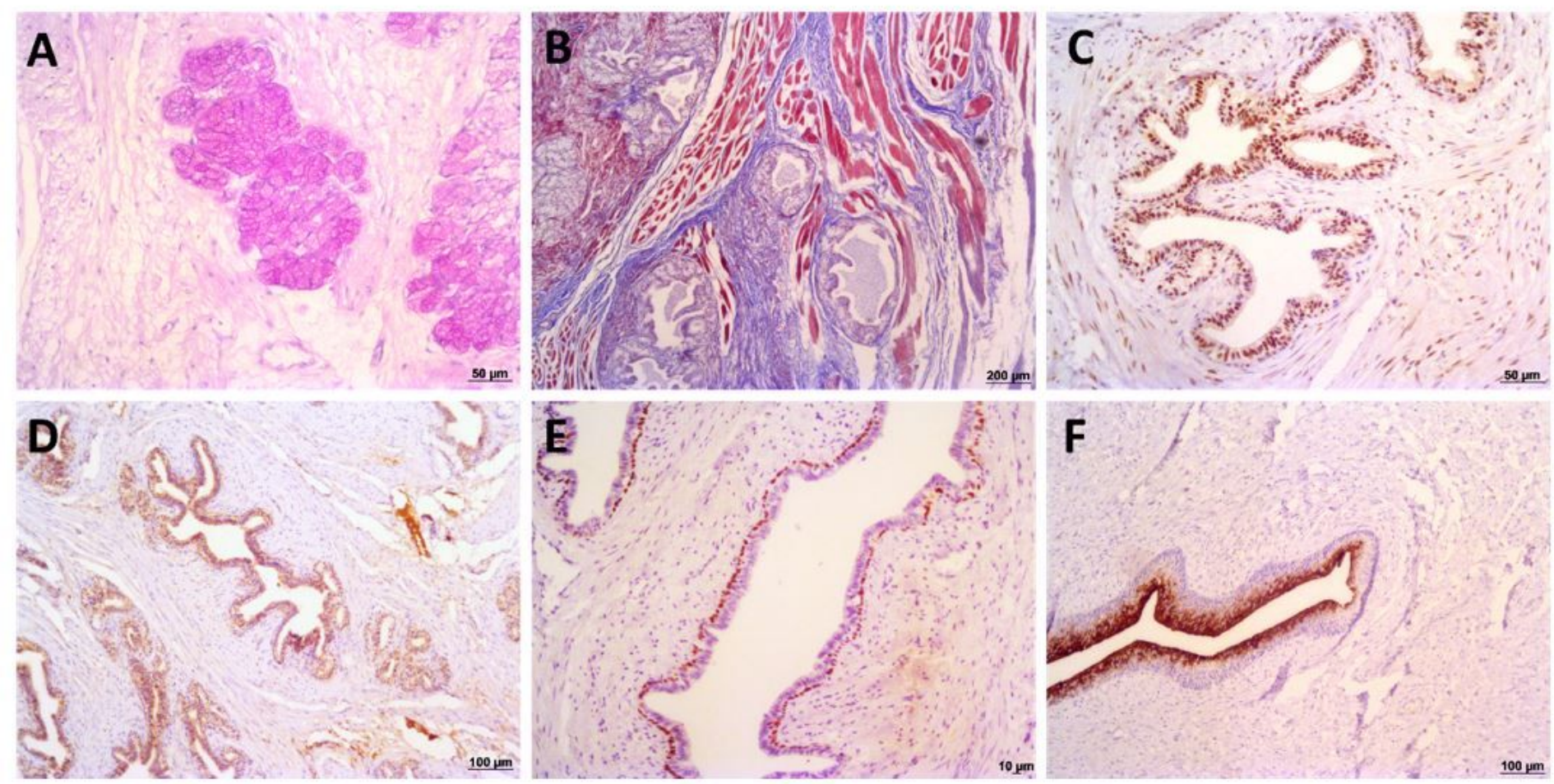

\section{Figure 4}

Histochemical and Immunohistochemical analysis of Giant Anteater prostate gland. A: Luminal cells from peripheral zone presenting PAS-rich secretory cells. B: Trichrome Masson staining presenting muscle fibers staining in red and collagen fibers stained in blue. It is possible to note a fibromuscular stroma in peripheral zone. C: Androgen receptor (AR) nuclear immunoexpression in prostatic epithelial cells. D: Prostatic Specific Antigen (PSA) staining in prostatic epithelial cells. E: p63 positive expression by basal prostatic cells. F: Uroplakin III immunexpression by urothelial superficial cells from prostatic urethra. 


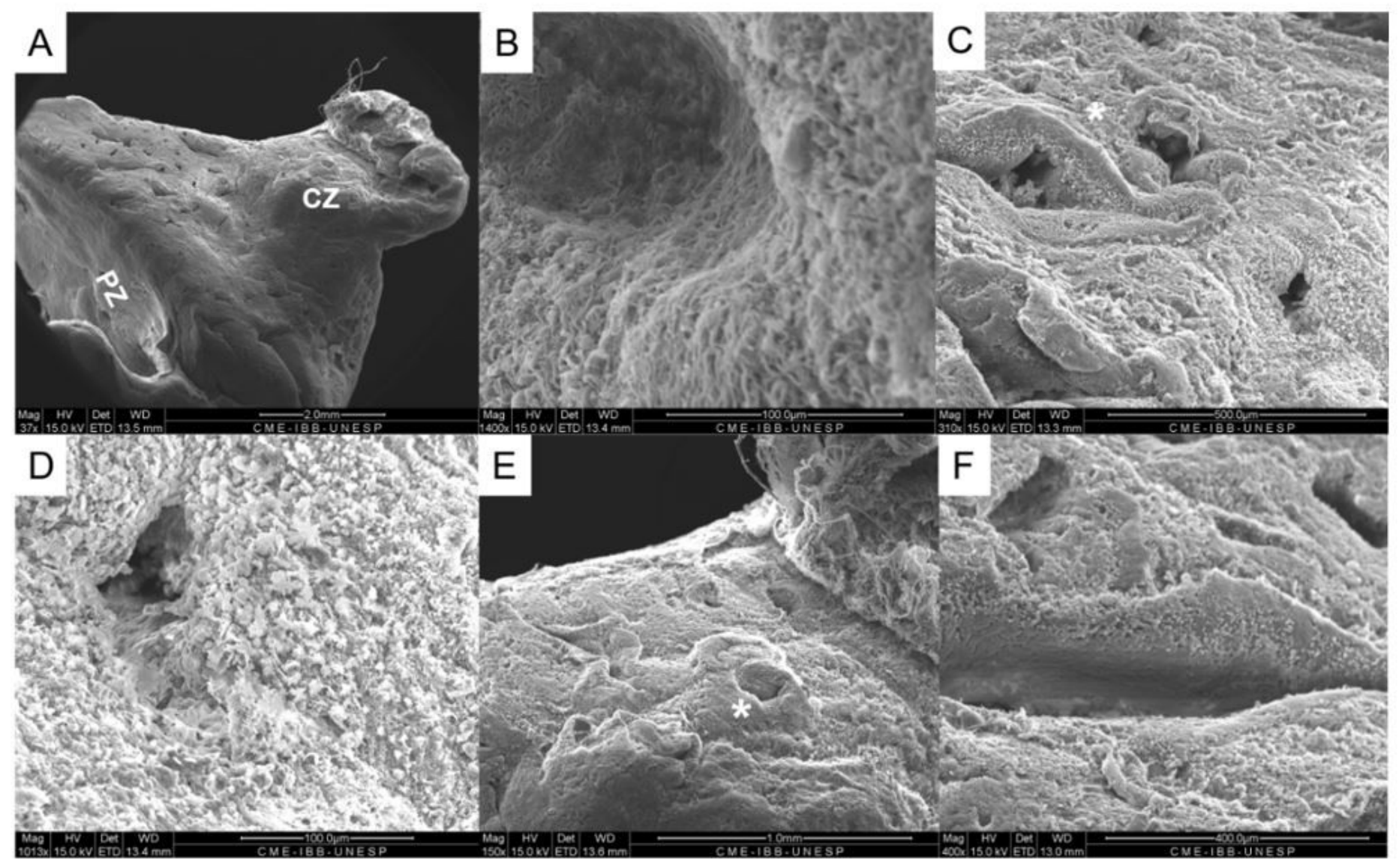

Figure 5

Scanning electronic microscopy. Ultrastructural lower magnification distinguishing the central and peripherals zones ( $\mathrm{ZC}$ to the central zone and $\mathrm{ZP}$ to the peripherical zone in picture $\mathrm{A}$ ). It is possible to visualize the capsule (B), the peripherical zone with the acinus (asterisk) (C), whose epithelium can be observed in higher magnification (D), the urethra prostatic in the central zone (asterisk) (E), surrounded by the prostatic ducts $(F)$. 